\title{
Uplink Interference Management in Massive MIMO Enabled Heterogeneous Cellular
} Networks

\author{
Anqi He, Lifeng Wang, Yue Chen, Kai-Kit Wong, and Maged Elkashlan
}

\begin{abstract}
In this letter, we exploit the implementation of uplink power control and cell range extension for interference coordination in a two-tier massive MIMO enabled heterogeneous cellular network. Our results show that the uplink performance in the massive MIMO macrocells can be significantly improved through uplink power control in the small cells, while more uplink transmissions in the macrocells have mild adverse effect on the uplink performance of the small cells. In addition, the uplink performance of the massive MIMO macrocells can be improved by expanding the small cell range when dozens of users are served in the macrocells.
\end{abstract}

Index Terms

Massive MIMO, heterogeneous cellular networks, uplink interference management.

A. He, Y. Chen, and M. Elkashlan are with the School of Electronic Engineering and Computer Science, Queen Mary University of London, London, UK. (Email: \{a.he, yue.chen, maged.elkashlan\}@qmul.ac.uk)

L. Wang, and K.-K. Wong are with the Department of Electronic and Electrical Engineering, University College London, London, UK (Email: \{lifeng.wang, kai-kit.wong\}@ucl.ac.uk). 


\section{INTRODUCTION}

Massive multiple input multiple output (MIMO) and heterogeneous cellular network (HCN) are two promising solutions for supporting high data traffic in 5G networks. Massive MIMO, as a large-scale multiuser MIMO transmission strategy, has the capability of communicating with dozens of users at the same time and frequency band [1]. In HCNs, small cell base stations are deployed on a large-scale, which allows more users to be served. As such, massive MIMO aided HCNs has recently attracted much attention $[2,3]$.

In the massive MIMO aided HCNs, downlink transmission has been examined by considering different performance metrics such as spectrum efficiency [2] or energy efficiency [3]. However, interference management is critically important for the uplink transmission, since more users served by the massive MIMO macrocells and large numbers of small cells results in more severe uplink interference. The work of [4] adjusted the maximum transmit power of femtocell users for interference mitigation in two-tier femtocell networks and two schemes were proposed to suppress the cross-tier interference under a fixed threshold and an adaptive threshold. The uplink power control under quality constraints for enhancing network performance in a massive MIMO multicell network was investigated in [5].

While the aforementioned literature laid a good foundation in understanding the uplink power control for coordinating interference in the HCNs or massive systems, interference management for a system which integrates both the two key technologies has not been conducted yet. As such, we propose a joint uplink power control and CRE scheme for uplink interference coordination in a two-tier massive MIMO aided HCN consisting of picocells and massive MIMO macrocells. Specifically, due to the massive MIMO array gains, the uplink/downlink imbalance is boosted in the massive MIMO aided HCNs. In light of this, CRE is employed to tackle the uplink/downlink imbalance problem. Since users in the massive MIMO macrocells are provided with large array gains, the level of their transmit power can be greatly lowered. Hence, we adopt the uplink power control in the picocells. We finally evaluate the performance of the proposed massive MIMO aided HCNs in terms of area spectrum efficiency.

\section{SYSTEM DESCRIPTION}

We consider the uplink transmission in a two-tier heterogeneous networks (HetNets), in which massive MIMO macrocells are overlaid with picocells. The locations of macrocell base 
stations (MBSs) are modeled following a homogeneous Poisson point process (PPP) $\Phi_{\mathrm{M}}$ with density $\lambda_{\mathrm{M}}$. The locations of picocell base stations (PBSs) are modeled following an independent homogeneous PPP $\Phi_{\mathrm{P}}$ with density $\lambda_{\mathrm{P}}$. Each macrocell $\mathrm{BS}$ is equipped with $N$ antennas and receives data streams from $S$ single-antenna users over the same time and frequency band, while each PBS equipped with one single antenna receives one data stream from a single-antenna user in each transmission. It is assumed that the density of users is much greater than that of BSs so that there always will be one active mobile user at each time slot in every small cell and multiple active mobile users in every macrocell. The zero-forcing beamforming (ZFBF) is employed to cancel the intra-cell interference at the MBS, and the ZFBF matrix at a MBS is $\mathbf{W}=\mathbf{G}\left(\mathbf{G}^{H} \mathbf{G}\right)^{-1}$ with the channel matrix $\mathbf{G}[6]$, where $H$ denotes the Hermitian transpose. All the channels undergo independent and identically distributed (i.i.d.) quasi-static Rayleigh fading.

\section{A. Uplink Power Control}

Owing to the benefits of massive MIMO such as large antenna array gain, users associated with the MBSs can use lower transmit power, hence we focus on the uplink power control in the picocells. Specifically, the open-loop uplink power control is applied in the picocells, and the transmit power for a user associated with the PBS is given by

$$
P_{\mathrm{u}, \mathrm{P}}=\min \left\{P_{\max }, P_{o} L\left(\left|X_{\kappa}\right|\right)^{-\eta}\right\}
$$

where $P_{\max }$ is the maximum transmit power, $P_{o}$ is the normalized power density, $L\left(\left|X_{\kappa}\right|\right)=$ $\beta\left|X_{\kappa}\right|^{-\alpha}$ is the path loss with the exponent $\alpha$, and $\beta$ is the frequency dependent constant value, $\left|X_{\kappa}\right|$ is the distance between the user and its associated PBS, $\eta \in[0,1]$ is the path loss compensation factor, which controls the picocell user's transmit power. Here $\eta=1$ represents that the path loss between a user and its serving PBS is fully compensated, and $\eta=0$ represents that there is no path loss compensation.

\section{B. User Association}

We consider the downlink-centric user association ${ }^{1}$. In the downlink, each MBS transmits $S$ user-streams with equal power assignment. As such, the long-term average downlink receive

\footnotetext{
${ }^{1}$ Although user association for the downlink and uplink can be decoupled in the conventional cellular networks, the main drawback for the decoupling is that channel reciprocity in massive MIMO systems will be lost [7].
} 
power $P_{\ell}^{\mathrm{r}}$ at a macrocell user (MUE) which is connected with the MBS $\ell\left(\ell \in \Phi_{\mathrm{M}}\right)$ is

$$
P_{\ell}^{\mathrm{r}}=G_{a} \frac{P_{\mathrm{M}}}{S} L\left(\left|X_{\ell}\right|\right)
$$

where $G_{a}$ is the array gain, $P_{\mathrm{M}}$ is the MBS's transmit power, $L\left(\left|X_{\ell}\right|\right)=\beta\left|X_{\ell}\right|^{-\alpha_{\mathrm{M}}}$ is the path loss function, $\left|X_{\ell}\right|$ is the distance, and $\alpha_{\mathrm{M}}$ is the path loss exponent. The array gain $G_{a}$ obtained by the ZFBF transmission is $N-S+1$ [2]. In the picocell, considering the effect of picocell range expansion, the long-term average downlink receive power $P_{\kappa}^{\mathrm{r}}$ at a picocell user (PUE) which is connected with the PBS $\kappa\left(\kappa \in \Phi_{\mathrm{P}}\right)$ is expressed as

$$
P_{\kappa}^{\mathrm{r}}=P_{\mathrm{P}} L\left(\left|X_{\kappa}\right|\right) B
$$

where $P_{\mathrm{P}}$ is the PBS's transmit power and $L\left(\left|X_{\kappa}\right|\right)=\beta\left(\left|X_{\kappa}\right|\right)^{-\alpha_{\mathrm{P}}}$ is the path loss function, and $B$ is the biasing factor, which is useful for offloading the data traffic to small cells in conventional HetNets [8].

\section{Uplink Channel Model}

We assume that a typical serving base station is located at the origin $o$. The receive signal-tointerference-plus-noise ratio (SINR) of a typical serving MBS at a random distance $\left|X_{o, \mathrm{M}}\right|$ from its intended MUE is given by

$$
\mathrm{SINR}_{\mathrm{M}}=\frac{P_{\mathrm{u}, \mathrm{M}} h_{o, \mathrm{M}} L\left(\left|X_{o, \mathrm{M}}\right|\right)}{I_{\mathrm{M}}+I_{\mathrm{P}}+\delta^{2}},
$$

where $I_{\mathrm{M}}=\sum_{i \in \Phi_{\mathrm{u}, \mathrm{M}} \backslash \mathrm{B}_{o, \mathrm{M}}} P_{\mathrm{u}, \mathrm{M}} h_{i, \mathrm{M}} L\left(\left|X_{i, \mathrm{M}}\right|\right)$ is the uplink intra-tier interference, $I_{\mathrm{P}}=$ $\sum_{j \in \Phi_{\mathrm{u}, \mathrm{P}}} P_{j, \mathrm{P}} h_{j, \mathrm{M}} L\left(\left|X_{j, \mathrm{M}}\right|\right)$ is the uplink inter-tier interference, $P_{\mathrm{u}, \mathrm{M}}$ and $P_{j, \mathrm{P}}$ are the transmit power of the MUE and PUE $j \in \Phi_{\mathrm{u}, \mathrm{P}}\left(\Phi_{\mathrm{u}, \mathrm{P}}\right.$ is the point process corresponding to the interfering PUEs), respectively, $h_{o, \mathrm{M}} \sim \Gamma(N-S+1,1)$ [6] is the small-scale fading channel power gain between the typical serving MBS and its intended user, $h_{i, \mathrm{M}} \sim \exp (1)$ and $\left|X_{i, \mathrm{M}}\right|$ are the smallscale fading interfering channel power gain and distance between the typical serving MBS and interfering MUE $i \in \Phi_{\mathrm{u}, \mathrm{M}} \backslash \mathrm{B}_{o, \mathrm{M}}\left(\Phi_{\mathrm{u}, \mathrm{M}} \backslash \mathrm{B}_{o, \mathrm{M}}\right.$ is the point process corresponding to the interfering MUEs), respectively, $h_{j, \mathrm{M}} \sim \exp (1)$ and $\left|X_{j, \mathrm{M}}\right|$ are the small-scale fading interfering channel power gain and distance between the typical serving MBS and interfering PUE $j$, respectively, and $\delta^{2}$ is the noise power. 


$$
\begin{aligned}
& f_{\left|X_{o, \mathrm{M}}\right|}(x)=\frac{2 \pi \lambda_{\mathrm{M}}}{\mathcal{A}_{\mathrm{M}}} x \exp \left\{-\pi \lambda_{\mathrm{M}} x^{2}-\pi \lambda_{\mathrm{P}}\left(\frac{P_{\mathrm{P}} B S x^{\alpha_{\mathrm{M}}}}{P_{\mathrm{M}}(N-S+1)}\right)^{2 / \alpha_{\mathrm{P}}}\right\} \\
& f_{\left|X_{o, \mathrm{P}}\right|}(x)=\frac{2 \pi \lambda_{\mathrm{P}}}{\mathcal{A}_{\mathrm{P}}} x \exp \left\{-\pi \lambda_{\mathrm{P}} x^{2}-\pi \lambda_{\mathrm{M}}\left(\frac{P_{\mathrm{M}}(N-S+1) x^{\alpha_{\mathrm{P}}}}{P_{\mathrm{P}} B S}\right)^{2 / \alpha_{\mathrm{M}}}\right\}
\end{aligned}
$$

where

$$
\begin{aligned}
& \mathcal{A}_{\mathrm{M}}=2 \pi \lambda_{\mathrm{M}} \int_{0}^{\infty} r \exp \left\{-\pi \lambda_{\mathrm{M}} r^{2}-\pi \lambda_{\mathrm{P}}\left(\frac{P_{\mathrm{P}} B S r^{\alpha_{M}}}{P_{M}(N-S+1)}\right)^{2 / \alpha_{\mathrm{P}}}\right\} d r \\
& \mathcal{A}_{\mathrm{P}}=2 \pi \lambda_{\mathrm{P}} \int_{0}^{\infty} r \exp \left\{-\pi \lambda_{\mathrm{P}} r^{2}-\pi \lambda_{\mathrm{M}}\left(\frac{P_{\mathrm{M}}(N-S+1) r^{\alpha_{\mathrm{P}}}}{P_{\mathrm{P}} B S}\right)^{2 / \alpha_{\mathrm{M}}}\right\} d r
\end{aligned}
$$

The SINR of a typical serving PBS at a random distance $\left|X_{o, \mathrm{P}}\right|$ from its intended user is given by

$$
\mathrm{SINR}_{\mathrm{P}}=\frac{P_{\mathrm{u}, \mathrm{P}} g_{o, \mathrm{P}} L\left(\left|X_{o, \mathrm{P}}\right|\right)}{J_{\mathrm{M}}+J_{\mathrm{P}}+\delta^{2}}
$$

where $J_{\mathrm{M}}=\sum_{i \in \Phi_{\mathrm{u}, \mathrm{M}}} P_{\mathrm{u}, \mathrm{M}} g_{i, \mathrm{P}} L\left(\left|X_{i, \mathrm{P}}\right|\right), J_{\mathrm{P}}=\sum_{j \in \Phi_{\mathrm{u}, \mathrm{P}} \backslash \mathrm{B}_{o, \mathrm{P}}} P_{j, \mathrm{P}} g_{j, \mathrm{P}} L\left(\left|X_{j, \mathrm{P}}\right|\right), g_{o, \mathrm{P}} \sim \exp (1)$ is the small-scale fading channel power gain between the typical serving PBS and its intended user, $g_{i, \mathrm{P}} \sim \exp (1)$ and $\left|X_{i, \mathrm{P}}\right|$ are the small-scale fading interfering channel power gain and distance between the typical serving PBS and interfering user $i \in \Phi_{\mathrm{u}, \mathrm{M}}$, respectively, $g_{j, \mathrm{P}} \sim \exp (1)$ and $\left|X_{j, \mathrm{P}}\right|$ are the small-scale fading interfering channel power gain and distance between the typical serving MBS and interfering user $j \in \Phi_{\mathrm{u}, \mathrm{P}} \backslash \mathrm{B}_{o, \mathrm{P}}$ (except the typical PUE), respectively.

\section{Area Uplink Spectrum EFFICIENCy}

In this section, we evaluate the area uplink spectrum efficiency for massive MIMO macrocell tier and picocell tier. The area uplink spectrum efficiency allows us to examine the impacts of different densities of picocells and massive MIMO macrocells on the uplink spectrum efficiency of different tiers.

Based on [8], we first obtain the probability density functions of the distance $\left|X_{o, \mathrm{M}}\right|$ between a typical MUE and its serving MBS $B_{o, \mathrm{M}}$ and the distance $\left|X_{o, \mathrm{P}}\right|$ between a typical PUE and its serving PBS $B_{o, P}$ as (6) and (7) at the top of the next page, respectively. Based on the distance statistic properties, we have the following two theorems. 


$$
\begin{aligned}
& \Xi_{1}(x)=\frac{P_{u, \mathrm{M}} \beta 2 \pi S \lambda_{\mathrm{M}}}{\alpha_{\mathrm{M}}-2} x^{2-\alpha_{\mathrm{M}}}, \\
& \Xi_{2}(x)=\frac{2 \pi \lambda_{\mathrm{P}} \beta}{\alpha_{\mathrm{M}}-2}\left(R_{\mathrm{M}}(x)\right)^{2-\alpha_{\mathrm{M}}}\left(P_{o} \beta^{-\eta} \int_{0}^{r_{o}} t^{\alpha_{\mathrm{P}} \eta} f_{\left|X_{o, \mathrm{P}}\right|}(t) d t+P_{\max } \int_{r_{o}}^{\infty} f_{\left|X_{o, \mathrm{P}}\right|}(t) d t\right)
\end{aligned}
$$

$$
\begin{aligned}
& F_{\left\{X_{o, \mathrm{P}}=x\right\}}(\gamma)=\exp \left\{-\frac{\delta^{2} x^{\alpha_{\mathrm{P}}} \gamma}{\beta P_{o, \mathrm{P}}}-S \lambda_{\mathrm{M}} 2 \pi \frac{x^{\alpha_{\mathrm{P}}} \gamma}{P_{o, \mathrm{P}}} P_{u, \mathrm{M}} \frac{\left(R_{\mathrm{P}}(x)\right)^{2-\alpha_{\mathrm{P}}}}{\alpha_{\mathrm{P}}-2}\right. \\
& { }_{2} F_{1}\left[\frac{\alpha_{\mathrm{P}}-2}{\alpha_{\mathrm{P}}}, 1 ; 2-\frac{2}{\alpha_{\mathrm{P}}} ;-\frac{x^{\alpha_{\mathrm{P}}} \gamma}{P_{o, \mathrm{P}}} P_{u, \mathrm{M}}\left(R_{\mathrm{P}}(x)\right)^{-\alpha_{\mathrm{P}}}\right]-\lambda_{\mathrm{P}} 2 \pi \frac{x^{\alpha_{\mathrm{P}}} \gamma}{P_{o, \mathrm{P}}} P_{o} \beta^{-\eta} \frac{x^{2-\alpha_{\mathrm{P}}}}{\alpha_{\mathrm{P}}-2} \int_{0}^{r_{o}} t^{\alpha_{\mathrm{P}} \eta} \\
& { }_{2} F_{1}\left[\frac{\alpha_{\mathrm{P}}-2}{\alpha_{\mathrm{P}}}, 1 ; 2-\frac{2}{\alpha_{\mathrm{P}}} ;-\frac{x^{\alpha_{\mathrm{P}}} \gamma}{P_{o, \mathrm{P}}} P_{o} \beta^{-\eta} t^{\alpha_{\mathrm{P}} \eta} x^{-\alpha_{\mathrm{P}}}\right] f_{\left|X_{o, \mathrm{P}}\right|}(t) d t \\
& \left.-\lambda_{\mathrm{P}} 2 \pi \frac{x^{\alpha_{\mathrm{P}}} \gamma}{P_{o, \mathrm{P}}} P_{\max } \frac{x^{2-\alpha_{\mathrm{P}}}}{\alpha_{\mathrm{P}}-2}{ }_{2} F_{1}\left[\frac{\alpha_{\mathrm{P}}-2}{\alpha_{\mathrm{P}}}, 1 ; 2-\frac{2}{\alpha_{\mathrm{P}}} ;-\frac{x^{\alpha_{\mathrm{P}}} \gamma}{P_{o, \mathrm{P}}} P_{\max } x^{-\alpha_{\mathrm{P}}}\right] \int_{r_{o}}^{\infty} f_{\left|X_{o, \mathrm{P}}\right|}(t) d t\right\}
\end{aligned}
$$

Theorem 1: The area uplink spectrum efficiency for the macrocell tier is lower bounded as

$$
\mathcal{R}_{\mathrm{M}}^{\mathrm{L}}=S \lambda_{\mathrm{M}} \log _{2}\left(1+e^{\Delta_{1}+\Delta_{2}}\right),
$$

where

$$
\begin{aligned}
\Delta_{1}= & \ln \left(P_{u, \mathrm{M}} \beta\right)+\psi(N-S+1) \\
& -\alpha_{\mathrm{M}} \int_{0}^{\infty} \ln (x) f_{\left|X_{o, \mathrm{M}}\right|}(x) d x,
\end{aligned}
$$

and

$$
\begin{array}{r}
\Delta_{2}=-\ln \left(\int_{0}^{\infty} \Xi_{1}(x) f_{\left|X_{o, \mathrm{M}}\right|}(x) d x+\right. \\
\left.\int_{0}^{\infty} \Xi_{2}(x) f_{\left|X_{o, \mathrm{M} \mid}\right|}(x) d x+\delta^{2}\right),
\end{array}
$$

where $\psi(\cdot)$ is the digamma function [9], $f_{\left|X_{o, \mathrm{M}}\right|}$ is given by (6), $\Xi_{1}(x)$ and $\Xi_{2}(x)$ are given by (10) and (11) in the following page, $R_{\mathrm{M}}(x)=\left(\frac{P_{\mathrm{P}} B S}{P_{\mathrm{M}}(N-S+1)}\right)^{1 / \alpha_{\mathrm{P}}} x^{\alpha_{\mathrm{M}} / \alpha_{\mathrm{P}}}$, and $r_{o}=\left(\frac{P_{\max }}{P_{o}}\right)^{1 /\left(\alpha_{\mathrm{P}} \eta\right)} \beta^{1 / \alpha_{\mathrm{P}}}$.

Proof: The proof is provided in Appendix A. 
Theorem 2: The area uplink spectrum efficiency for the picocell tier is given by

$$
\mathcal{R}_{\mathrm{P}}=\frac{\lambda_{\mathrm{P}}}{\ln 2} \int_{0}^{\infty} \frac{F_{\mathrm{cov}}^{\mathrm{P}}(\gamma)}{1+\gamma} d \gamma,
$$

where $F_{\mathrm{cov}}^{\mathrm{P}}(\gamma)=\int_{0}^{\infty} F_{\left\{X_{o, \mathrm{P}}=x\right\}}(\gamma) f_{\left|X_{o, \mathrm{P}}\right|}(x) d x$ is the complementary distribution function $(\mathrm{CCDF})$ of $\operatorname{SINR}_{\mathrm{P}}$, here $f_{\left|X_{o, \mathrm{P}}\right|}(x)$ is given by (7) and $F_{\left\{X_{o, \mathrm{P}}=x\right\}}(\gamma)$ is given by (12), in which $P_{o, \mathrm{P}}=\min \left\{P_{\max }, P_{o}\left(\beta x^{-\alpha_{\mathrm{P}}}\right)^{-\eta}\right\}, R_{\mathrm{P}}(x)=\left(\frac{P_{\mathrm{M}}(N-S+1)}{P_{\mathrm{P}} B S}\right)^{1 / \alpha_{\mathrm{M}}} x^{\alpha_{\mathrm{P}} / \alpha_{\mathrm{M}}},{ }_{2} F_{1}[\cdot, \cdot ; \cdot ; \cdot]$ is the Gauss hypergeometric function [10, (9.142)], and $\mathbf{1}(A)$ is the indicator function that returns one if the condition $A$ is satisfied. The proof of (12) can be easily provided by following the similar approach in [8].

\section{NuMERICAL RESUlts}

In this section, numerical results are presented to evaluate the area uplink spectrum efficiency of MUE and PUE in the massive MIMO aided HCN. The density of MBSs is $\lambda_{\mathrm{M}}=$ $\left(500^{2} \times \pi\right)^{-1} \mathrm{~m}^{-2}$ in a circular region with radius $1 \times 10^{4} \mathrm{~m}$. Each MBS is equipped with $N=200$ antennas. Such a network is assumed to operate at a carrier frequency of $1 \mathrm{GHz}$, the path loss exponents are $\alpha_{\mathrm{M}}=3.5$ and $\alpha_{\mathrm{P}}=4$, the MBS transmit power is $P_{\mathrm{M}}=43 \mathrm{dBm}$, the PBS transmit power is $P_{\mathrm{R}}=30 \mathrm{dBm}$, the MUE transmit power is set as $15 \mathrm{dBm}$, and the maximum PUE transmit power is $P_{\max }=23 \mathrm{dBm}$. The power density is $P_{o}=-80 \mathrm{dBm}$ [11]. In the figures, Monte Carlo (MC) simulated values of the uplink spectrum efficiency marked by 'o' are numerically obtained to validate the analysis.

Fig. 1 shows the area uplink spectrum efficiency versus density ratio between PBS and MBS. Each MBS is equipped with $N=400$ antennas, the number of users served in each macrocell is $S=25$ and the biasing factor is $B=3 \mathrm{~dB}$. We see that the lower bound curves for area uplink spectrum efficiency of the macrocell tier obtained from Theorem 1 can efficiently predict the performance behavior. Uplink power control applied in the picocells can significantly affect the area uplink spectrum efficiency of the macrocell tier and picocell tier. Specifically, when the pathloss compensation factor is lower, uplink performance in the macrocells is enhanced, due to the fact that macrocells experience less interference from the uplink transmissions in the picocells. Although users in the picocells can use the maximum transmit power $P_{\max }=23$ $\mathrm{dBm}$ when they are not close to their serving PBSs, the area uplink spectrum efficiency of the picocell tier is declined. The reason is that the transmit power of the users who are very close 


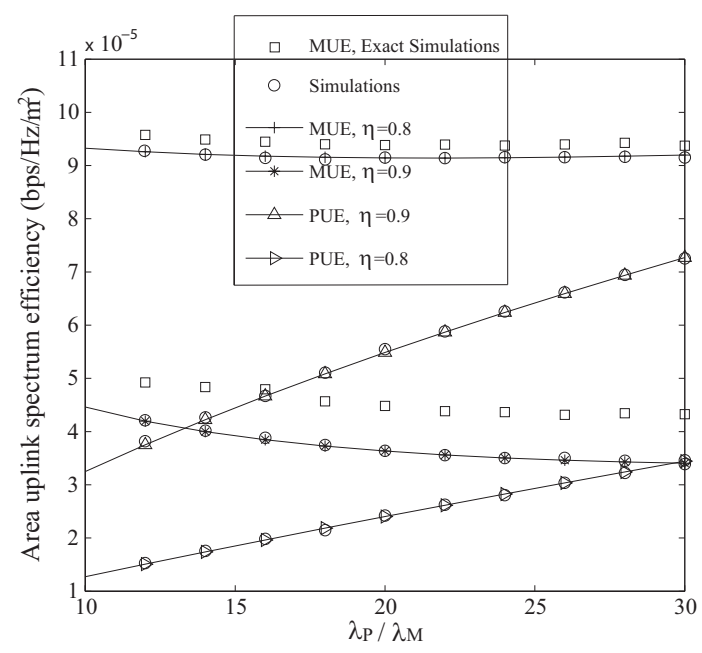

Fig. 1. The effect of power control.

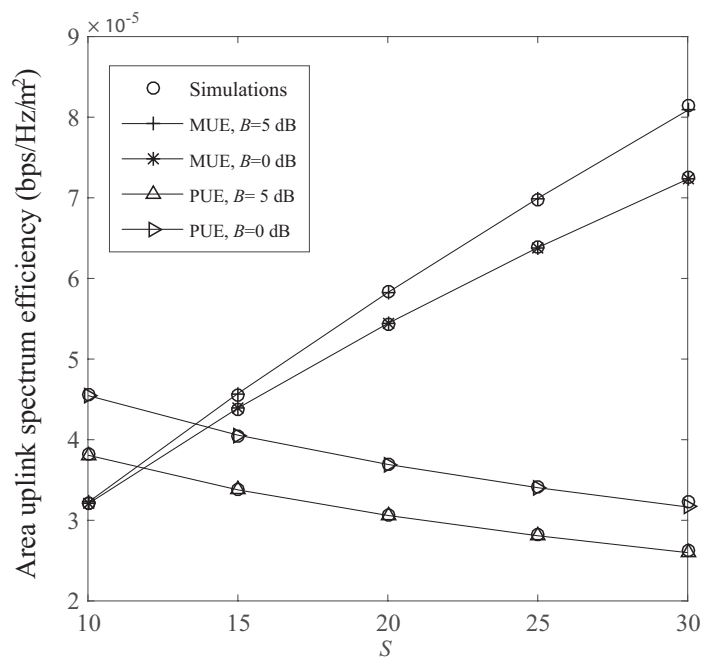

Fig. 2. The effect of cell range expansion (CRE).

to their serving PBSs is controlled, and their achievable uplink spectrum efficiency decreases significantly. Deploying more PBSs improves the area uplink spectrum efficiency of picocell tier, however, the uplink performance of the macrocell tier decreases due to more uplink interference from the picocell tier.

Fig. 2 shows the the area uplink spectrum efficiency versus number of users served by each MBS. Each MBS is equipped with $N=200$ antennas, the density of PBSs is $\lambda_{\mathrm{P}}=30 \times \lambda_{\mathrm{M}}$, 
and the power control compensation factor $\eta=0.8$. We find that CRE has a substantial effect on the area uplink spectrum efficiency of the macrocell tier, when more users per marcocell are served. However, the effect of CRE on the uplink performance of picocell tier is not significant, since users in the picocells are allowed to use large transmit power (the maximum $P_{\max }=23$ $\mathrm{dBm}$ ) when they are comparatively far from their serving PBSs. Obviously, serving more users increases the area spectrum efficiency of the macrocell tier due to more multiplexing gains, and more uplink transmissions in the macrocells result in a mild decrease in the area uplink spectrum efficiency of the picocell tier, since interference from MUEs increases.

\section{CONCLUSION}

We considered uplink interference mitigation in the massive MIMO aided heterogeneous cellular networks. By jointly adopting uplink power control and cell range expansion, our results confirmed that uplink inter-tier interference can be effectively coordinated.

\section{APPENDIX A: A PROOF OF THEOREM 1}

Based on (4), the area uplink spectrum efficiency is calculated as

$$
\mathcal{C}_{\mathrm{M}}=S \lambda_{\mathrm{M}} \underbrace{\mathrm{E}\left\{\log _{2}\left(1+\mathrm{SINR}_{\mathrm{M}}\right)\right\}}_{\Theta_{1}} \text {. }
$$

By using Jensen's inequality, a lower bound for $\Theta_{1}$ is

$$
\Theta_{1}^{L}=\log _{2}\left(1+e^{Z_{1}+Z_{2}}\right)
$$

where

$$
\left\{\begin{array}{l}
Z_{1}=\mathrm{E}\left\{\ln \left(P_{u, M} h_{o, M} \beta\left|X_{o, M}\right|^{-\alpha_{M}}\right)\right\}, \\
Z_{2}=\mathrm{E}\left\{\ln \left(\frac{1}{I_{M}+I_{P}+\delta^{2}}\right)\right\} .
\end{array}\right.
$$

We first calculate $Z_{1}$ as

$$
Z_{1}=\ln \left(P_{u, \mathrm{M}} \beta\right)+\mathrm{E}\left\{\ln \left(h_{o, \mathrm{M}}\right)\right\}-\alpha_{\mathrm{M}} \mathrm{E}\left\{\ln \left|X_{o, \mathrm{M}}\right|\right\}
$$

Considering that $h_{o, \mathrm{M}} \sim \Gamma(N-S+1), \mathrm{E}\left\{\ln \left(h_{o, M}\right)\right\}$ is calculate as $\mathrm{E}\left\{\ln \left(h_{o, \mathrm{M}}\right)\right\}=\psi(N-S+1)$. We next derive $\mathrm{E}\left\{\ln \left|X_{o, \mathrm{M}}\right|\right\}$ as

$$
\mathrm{E}\left\{\ln \left|X_{o, \mathrm{M}}\right|\right\}=\int_{0}^{\infty} \ln (x) f_{\left|X_{o, \mathrm{M}}\right|}(x) d x
$$


By using Jensen's inequality, we derive the lower bound on the $Z_{2}$ as

$$
Z_{2}>\overline{Z_{2}}=\ln \left(\frac{1}{\mathrm{E}\left\{I_{\mathrm{M}}\right\}+\mathrm{E}\left\{I_{\mathrm{P}}\right\}+\delta^{2}}\right)
$$

Then, we have

$$
\begin{aligned}
& \mathrm{E}\left\{I_{\mathrm{M}}\right\}=\int_{0}^{\infty} \mathrm{E}\left\{I_{\mathrm{M}}|| X_{o, \mathrm{M}} \mid=x\right\} f_{\left|X_{o, \mathrm{M}}\right|}(x) d x \\
& \stackrel{(a)}{=} \int_{0}^{\infty}\left(P_{u, \mathrm{M}} \beta 2 \pi S \lambda_{\mathrm{M}} \int_{x}^{\infty} r^{1-\alpha_{\mathrm{M}}} d r\right) f_{\left|X_{o, \mathrm{M}}\right|}(x) d x
\end{aligned}
$$

where (a) results from using the Campbell's theorem [12]. Similarly, $\mathrm{E}\left\{I_{\mathrm{P}}\right\}$ can also be obtained. Substituting (A.3) and (A.2) into (A.1), we get (13).

\section{REFERENCES}

[1] H. Q. Ngo, E. G. Larsson, and T. L. Marzetta, "Energy and spectral efficiency of very large multiuser MIMO systems," IEEE Trans. Commun., vol. 61, no. 4, pp. 1436-1449, 2013.

[2] Y. Xu and S. Mao, "User association in massive MIMO hetnets," IEEE System Journal, pp. 1-13, 2015.

[3] D. Liu et al., "Distributed energy efficient fair user association in massive MIMO enabled HetNets," IEEE Commun. Lett., vol. 19, no. 10, pp. 1770-1773, 2015.

[4] H.-S. Jo, C. Mun, J. Moon, and J.-G. Yook, "Interference mitigation using uplink power control for two-tier femtocell networks," IEEE Trans. Wireless Commun., vol. 8, no. 10, pp. 4906-4910, 2009.

[5] H. Yang and T. L. Marzetta, "On existence of power controls for massive MIMO," in IEEE Int. Symposium Inf. Theory (ISIT), 2015, pp. 2608-2612.

[6] K. Hosseini, W. Yu, and R. S. Adve, "Large-scale MIMO versus network MIMO for multicell interference mitigation," IEEE J. Sel. Topics Signal Process., vol. 8, no. 5, pp. 930-941, Oct. 2014.

[7] F. Boccardi et al., "Why to decouple the uplink and downlink in cellular networks and how to do it," arXiv preprint arXiv:1503.06746, Mar. 2015.

[8] H.-S. Jo, Y. J. Sang, P. Xia, and J. Andrews, "Heterogeneous cellular networks with flexible cell association: A comprehensive downlink sinr analysis," IEEE Trans. Wireless Commun., vol. 11, no. 10, pp. 3484-3495, October 2012.

[9] M. Abramowitz and I. A. Stegun, Handbook of Mathematical Functions with Formulas, Graphs, and Mathematical Tables, 9th ed. New York: Dover Publications, 1970.

[10] I. S. Gradshteyn and I. M. Ryzhik, Table of Integrals, Series and Products, 7th ed. San Diego, C.A.: Academic Press, 2007.

[11] K. Safjan et al., "Open loop power control parameter settings impact on lte hetnet uplink performance," in IEEE Int. Conf. Commun. (ICC) Workshops, 2013, pp. 1134-1138.

[12] F. Baccelli and B. Błaszczyszyn, Stochastic Geometry and Wireless Networks, Volume I: Theory. Now Publishers Inc. Hanover, MA, USA, 2009. 